\title{
Lyapunov exponent evaluation of a digital watermarking scheme proven to be secure
}

\author{
Jacques M. Bahi, Nicolas Friot, and Christophe Guyeux* \\ Computer science laboratory DISC \\ FEMTO-ST Institute, UMR 6174 CNRS \\ University of Franche-Comté, Besançon, France \\ \{jacques.bahi, nicolas.friot, christophe.guyeux $\} @$ femto-st.fr \\ * Authors in alphabetic order
}

August 9, 2021

\begin{abstract}
In our previous researches, a new digital watermarking scheme based on chaotic iterations has been introduced. This scheme was both stegosecure and topologically secure. The stego-security is to face an attacker in the "watermark only attack" category, whereas the topological security concerns other categories of attacks. Its Lyapunov exponent is evaluated here, to quantify the chaos generated by this scheme.
\end{abstract}

Keywords: Lyapunov exponent; Information hiding; Security; Chaotic iterations; Digital Watermarking.

\section{Introduction}

It currently exists only three data hiding schemes being both stego-secure and topologically secure. The first one is the "Natural Watermarking" with parameter $\eta=1$ [4]. The two others are based on chaotic iterations. The first of them is a one bit watermarking scheme 17, 2, whereas the last one allows steganographic operations [6]. In order to enlarge the knowledge about the security of these processes, the Lyapunov exponent of the digital watermarking scheme based on chaotic iterations is evaluated here.

This document is organized as follows. In Section 2, some basic reminders are given. The semiconjugacy allowing the exponent evaluation is described in Sect. 3. In the next one, the exponent is evaluated. This paper ends by a conclusion section where our contribution is summarized. 


\section{Basic Reminders}

\subsection{Chaotic Iterations and Watermarking Scheme}

Let us consider a system with a finite number $\mathbf{N} \in \mathbb{N}^{*}$ of cells, so that each cell has a boolean state. A sequence which elements belong into $\llbracket 1 ; \mathrm{N} \rrbracket$ is a strategy. Finally, the set of all strategies is denoted by $\llbracket 1, \mathrm{~N} \rrbracket^{\mathbb{N}}$. Let $S^{n}$ denotes the $n^{t h}$ term of a sequence $S$, and $V_{i}$ the $i^{\text {th }}$ component of a vector $V$.

Definition 1 The set $\mathbb{B}$ denoting $\{0,1\}$, let $f: \mathbb{B}^{N} \longrightarrow \mathbb{B}^{N}$ be a function and $S \in \llbracket 1, \mathrm{~N} \rrbracket^{\mathbb{N}}$. The chaotic iterations are defined by $x^{0} \in \mathbb{B}^{\mathrm{N}}$ and

$$
\forall n \in \mathbb{N}^{*}, \forall i \in \llbracket 1 ; \mathrm{N} \rrbracket, x_{i}^{n}= \begin{cases}x_{i}^{n-1} & \text { if } S^{n} \neq i \\ \left(f\left(x^{n-1}\right)\right)_{S^{n}} & \text { if } S^{n}=i .\end{cases}
$$

In other words, at the $n^{\text {th }}$ iteration, only the $S^{n}$-th cell is "iterated". Let us now recall how to define a suitable metric space where chaotic iterations are continuous 3 .

Let $\delta$ be the discrete boolean metric, $\delta(x, y)=0 \Leftrightarrow x=y$. Given a function $f$, define the function:

$$
\begin{aligned}
F_{f}: & \llbracket 1 ; \mathrm{N} \rrbracket \times \mathbb{B}^{\mathrm{N}} \longrightarrow \mathbb{B}^{\mathrm{N}} \\
& (k, E) \longmapsto\left(E_{j} . \delta(k, j)+f(E)_{k} . \overline{\delta(k, j)}\right)_{j \in \llbracket 1 ; \mathrm{N} \rrbracket}
\end{aligned}
$$

Consider the phase space $\mathcal{X}=\llbracket 1 ; \mathrm{N} \rrbracket^{\mathbb{N}} \times \mathbb{B}^{\mathrm{N}}$, and the map defined on $\mathcal{X}$ by:

$$
G_{f}(S, E)=\left(\sigma(S), F_{f}(i(S), E)\right),
$$

where $\sigma:\left(S^{n}\right)_{n \in \mathbb{N}} \in \llbracket 1, \mathbb{N} \rrbracket^{\mathbb{N}} \longrightarrow\left(S^{n+1}\right)_{n \in \mathbb{N}} \in \llbracket 1, \mathbb{N} \rrbracket^{\mathbb{N}}$ and $i:\left(S^{n}\right)_{n \in \mathbb{N}} \in$ $\llbracket 1, \mathbb{N} \rrbracket^{\mathbb{N}} \longrightarrow S^{0} \in \llbracket 1, \mathbb{N} \rrbracket$ are respectively the shift and the initial functions. Then chaotic iterations can be described by the following discreet dynamical system:

$$
\left\{\begin{array}{l}
X^{0} \in \mathcal{X} \\
X^{k+1}=G_{f}\left(X^{k}\right)
\end{array}\right.
$$

To study whether this dynamical system is chaotic [5], a distance between $X=(S, E)$ and $Y=(\breve{S}, \check{E}) \in \mathcal{X}$ has been introduced in [3] as follows: $d(X, Y)=$ $d_{e}(E, \check{E})+d_{s}(S, \check{S})$, where:

$$
d_{e}(E, \check{E})=\sum_{k=1}^{\mathrm{N}} \delta\left(E_{k}, \check{E}_{k}\right) \text { and } d_{s}(S, \check{S})=\frac{9}{\mathrm{~N}} \sum_{k=1}^{\infty} \frac{\left|S^{k}-\check{S}^{k}\right|}{10^{k}} .
$$

This distance has been introduced to satisfy the following requirements. If the floor value $\lfloor d(X, Y)\rfloor$ is equal to $n$, then the states $E$ and $\check{E}$ differ in $n$ cells. In addition, its floating part is less than $10^{-k}$ if and only if the first $k$ terms of the two strategies are equal. Moreover, if the $k^{t h}$ digit is nonzero, then the $k^{\text {th }}$ terms of the two strategies are different. With this metric, and the boolean vectorial negation $f_{0}$, it has been proven in 3 ] that, 
Theorem $1 G_{f_{0}}$ is continuous and chaotic in $(\mathcal{X}, d)$.

The digital watermarking scheme proposed in [7, 2] is simply the iterations of this dynamical system on the least significant coefficients of the considered media. Each property exhibited by the dynamical system will then be possessed too by the watermarking scheme. For further explanations, see [7, 2].

\subsection{The Lyapunov Exponent}

Some dynamical systems are very sensitive to small changes in their initial condition, which is illustrated by both the constants of sensitivity to initial conditions and of expansivity [3. However, these variations can quickly take enormous proportions, grow exponentially, and none of these constants can illustrate that. Alexander Lyapunov has examined this phenomenon and introduced an exponent that measures the rate at which these small variations can grow:

Definition 2 Given $f: \mathbb{R} \longrightarrow \mathbb{R}$, the Lyapunov exponent of the system composed by $x^{0} \in \mathbb{R}$ and $x^{n+1}=f\left(x^{n}\right)$ is defined by $\lambda\left(x_{0}\right)=\lim _{n \rightarrow+\infty} \frac{1}{n} \sum_{i=1}^{n} \ln \left|f^{\prime}\left(x^{i-1}\right)\right|$.

Consider a dynamic system with an infinitesimal error on the initial condition $x_{0}$. When the Lyapunov exponent is positive, this error will increase (situation of chaos), whereas it will decrease if $\lambda\left(x_{0}\right) \leqslant 0$.

Example 1 The Lyapunov exponent of the logistic map [1] becomes positive for $\mu>3,54$, but it is always smaller than 1 . The tent map [9] and the doubling map of the circle [8] have a Lyapunov exponent equal to $\ln (2)$.

To evaluate the Lyapunov exponent of our digital watermarking scheme, chaotic iterations must be described by a differentiable function on $\mathbb{R}$. To do so, a topological semiconjugacy between the phase space $\mathcal{X}$ and $\mathbb{R}$ must be written.

\section{A Topological Semiconjugacy}

\subsection{The Phase Space is an Interval of the Real Line}

\subsubsection{Toward a Topological Semiconjugacy}

We show, by using a topological semiconjugacy, that chaotic iterations on $\mathcal{X}$ can be described as iterations on a real interval. To do so, some notations and terminologies must be introduced.

Let $\mathcal{S}_{\mathrm{N}}=\llbracket 1 ; \mathrm{N} \rrbracket^{\mathbb{N}}$ be the set of sequences belonging into $\llbracket 1 ; \mathrm{N} \rrbracket$ and $\mathcal{X}_{\mathrm{N}}=$ $\mathcal{S}_{\mathrm{N}} \times \mathbb{B}^{\mathrm{N}}$. In what follows and for easy understanding, we will assume that $N=10$. However, an equivalent formulation of the following can be easily obtained by replacing the base 10 by any base $\mathrm{N}$. 
Definition 3 The function $\varphi: \mathcal{S}_{10} \times \mathbb{B}^{10} \rightarrow\left[0,2^{10}[\right.$ is defined by:

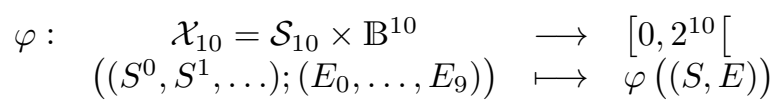

where $(S, E)=\left(\left(S^{0}, S^{1}, \ldots\right) ;\left(E_{0}, \ldots, E_{9}\right)\right)$, and $\varphi((S, E))$ is the real number:

- whose integral part $e$ is $\sum_{k=0}^{9} 2^{9-k} E_{k}$, that is, the binary digits of $e$ are $E_{0} E_{1} \ldots E_{9}$.

- whose decimal part $s$ is equal to $s=0, S^{0} S^{1} S^{2} \ldots=\sum_{k=1}^{+\infty} 10^{-k} S^{k-1}$.

$\varphi$ realizes the association between a point of $\mathcal{X}_{10}$ and a real number into $\left[0,2^{10}\left[\right.\right.$. We must now translate the digital watermarking process $G_{f_{0}}$ based on chaotic iterations on this real interval. To do so, two intermediate functions over $\left[0,2^{10}[\right.$ denoted by $e$ and $s$ must be introduced:

Definition 4 Let $x \in\left[0,2^{10}[\right.$ and:

- $e_{0}, \ldots, e_{9}$ the binary digits of the integral part of $x:\lfloor x\rfloor=\sum_{k=0}^{9} 2^{9-k} e_{k}$.

- $\left(s^{k}\right)_{k \in \mathbb{N}}$ the digits of $x$, where the chosen decimal decomposition of $x$ is the one that does not have an infinite number of 9: $x=\lfloor x\rfloor+\sum_{k=0}^{+\infty} s^{k} 10^{-k-1}$.

$e$ and $s$ are thus defined as follows:

$$
x \longmapsto\left(e_{0}, \ldots, e_{9}\right)
$$

and

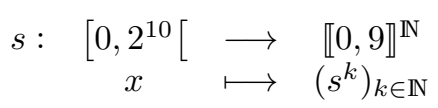

We are now able to define the function $g$, whose goal is to translate the chaotic iterations $G_{f_{0}}$ on an interval of $\mathbb{R}$.

Definition $5 \mathrm{~g}:\left[0,2^{10}\left[\longrightarrow\left[0,2^{10}[\right.\right.\right.$ is by definition such that $\mathrm{g}(\mathrm{x})$ is the real number of $\left[0,2^{10}[\right.$ defined bellow:

- its integral part has a binary decomposition equal to $e_{0}^{\prime}, \ldots, e_{9}^{\prime}$, with:

$$
e_{i}^{\prime}= \begin{cases}e(x)_{i} & \text { if } i \neq s^{0} \\ e(x)_{i}+1(\bmod 2) & \text { if } i=s^{0}\end{cases}
$$

- whose decimal part is $s(x)^{1}, s(x)^{2}, \ldots$ 
In other words, if $x=\sum_{k=0}^{9} 2^{9-k} e_{k}+\sum_{k=0}^{+\infty} s^{k} 10^{-k-1}$, then:

$$
g(x)=\sum_{k=0}^{9} 2^{9-k}\left(e_{k}+\delta\left(k, s^{0}\right)(\bmod 2)\right)+\sum_{k=0}^{+\infty} s^{k+1} 10^{-k-1} .
$$

\subsubsection{Defining a Metric on $\left[0,2^{10}[\right.$}

Numerous metrics can be defined on the set $\left[0,2^{10}[\right.$, the most usual one being the Euclidian distance $\Delta(x, y)=|y-x|^{2}$. This Euclidian distance does not reproduce exactly the notion of proximity induced by our first distance $d$ on $\mathcal{X}$. Indeed $d$ is richer than $\Delta$. This is the reason why we have to introduce the following metric:

Definition 6 Given $x, y \in\left[0,2^{10}\left[, D\right.\right.$ denotes the function from $\left[0,2^{10}\left[^{2}\right.\right.$ to $\mathbb{R}^{+}$defined by: $D(x, y)=D_{e}(e(x), e(y))+D_{s}(s(x), s(y))$, where:

$$
D_{e}(e, \check{e})=\sum_{k=0}^{9} \delta\left(e_{k}, \check{e}_{k}\right), \quad \text { and } D_{s}(s, \check{s})=\sum_{k=1}^{\infty} \frac{\left|s^{k}-\check{s}^{k}\right|}{10^{k}} \text {. }
$$

Proposition $1 D$ is a distance on $\left[0,2^{10}[\right.$.

Proof The three axioms defining a distance must be checked.

- $D \geqslant 0$, because everything is positive in its definition. If $D(x, y)=0$, then $D_{e}(x, y)=0$, so the integral parts of $x$ and $y$ are equal (they have the same binary decomposition). Additionally, $D_{s}(x, y)=0$, then $\forall k \in$ $\mathbb{N}^{*}, s(x)^{k}=s(y)^{k}$. In other words, $x$ and $y$ have the same $k$-th decimal digit, $\forall k \in \mathbb{N}^{*}$. And so $x=y$.

- $D(x, y)=D(y, x)$.

- Finally, the triangular inequality is obtained due to the fact that both $\delta$ and $|x-y|$ satisfy it.

The convergence of sequences according to $D$ is not the same than the usual convergence related to the Euclidian metric. For instance, if $x^{n} \rightarrow x$ according to $D$, then necessarily the integral part of each $x^{n}$ is equal to the integral part of $x$ (at least after a given threshold), and the decimal part of $x^{n}$ corresponds to the one of $x$ "as far as required". To illustrate this fact, a comparison between $D$ and the Euclidian distance is given Figure 1. These illustrations show that $D$ is richer and more refined than the Euclidian distance, and thus is more precise. 


\subsubsection{The Semiconjugacy}

It is now possible to define a topological semiconjugacy between $\mathcal{X}$ and an interval of $\mathbb{R}$ :

Theorem 2 Chaotic iterations on the phase space $\mathcal{X}$ are simple iterations on $\mathbb{R}$, which is illustrated by the semiconjugacy given bellow:

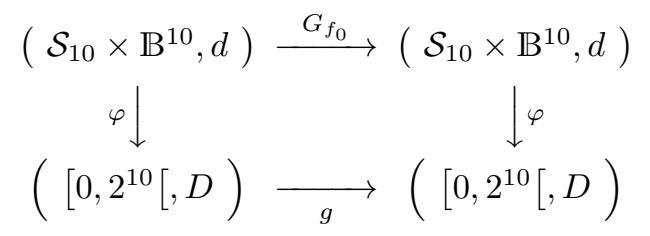

Proof $\varphi$ has been constructed in order to be continuous and onto.

In other words, $\mathcal{X}$ is approximately equal to $\left[0,2^{\mathrm{N}}[\right.$.

\subsection{Chaotic Iterations Described as a Real Function}

It can be remarked that the function $g$ is a piecewise linear function: it is linear on each interval having the form $\left[\frac{n}{10}, \frac{n+1}{10}\left[, n \in \llbracket 0 ; 2^{10} \times 10 \rrbracket\right.\right.$ and its slope is equal to 10. Let us justify these claims:

Proposition 2 Chaotic iterations $g$ defined on $\mathbb{R}$ have derivatives of all orders on $\left[0,2^{10}\left[\right.\right.$, except on the 10241 points in I defined by $\left\{\frac{n}{10} / n \in \llbracket 0 ; 2^{10} \times 10 \rrbracket\right\}$.

Furthermore, on each interval of the form $\left[\frac{n}{10}, \frac{n+1}{10}\left[\right.\right.$, with $n \in \llbracket 0 ; 2^{10} \times 10 \rrbracket$, $g$ is a linear function, having a slope equal to $10: \forall x \notin I, g^{\prime}(x)=10$.

Proof Let $I_{n}=\left[\frac{n}{10}, \frac{n+1}{10}\left[\right.\right.$, with $n \in \llbracket 0 ; 2^{10} \times 10 \rrbracket$. All the points of $I_{n}$ have the same integral part $e$ and the same decimal part $s^{0}$ : on the set $I_{n}$, functions $e(x)$ and $x \mapsto s(x)^{0}$ of Definition 4 only depend on $n$. So all the images $g(x)$ of these points $x$ :

- Have the same integral part, which is $e$, except probably the bit number $s^{0}$. In other words, this integer has approximately the same binary decomposition than $e$, the sole exception being the digit $s^{0}$ (this number is then either $e+2^{10-s^{0}}$ or $e-2^{10-s^{0}}$, depending on the parity of $s^{0}$, i.e., it is equal to $\left.e+(-1)^{s^{0}} \times 2^{10-s^{0}}\right)$.

- A shift to the left has been applied to the decimal part $y$, losing by doing so the common first digit $s^{0}$. In other words, $y$ has been mapped into $10 \times y-s^{0}$. 
To sum up, the action of $g$ on the points of $I$ is as follows: first, make a multiplication by 10 , and second, add the same constant to each term, which is $\frac{1}{10}\left(e+(-1)^{s^{0}} \times 2^{10-s^{0}}\right)-s^{0}$.

Remark 1 Finally, chaotic iterations used in our watermarking scheme are elements of the large family of functions that are both chaotic and piecewise linear (like the tent map 9 ).

We are now able to evaluate the Lyapunov exponent of our digital watermarking scheme based on chaotic iterations, which is now described by the iterations on $\mathbb{R}$ of the $g$ function introduced in Definition 5 .

\section{Evaluation of the Lyapunov Exponent}

Let $\mathcal{L}=\left\{x^{0} \in\left[0,2^{10}\left[/ \forall n \in \mathbb{N}, x^{n} \notin I\right\}\right.\right.$, where $I$ is the set of points in the real interval where $g$ is not differentiable (as it is explained in Proposition 2 ). Then,

Theorem $3 \forall x^{0} \in \mathcal{L}$, the Lyapunov exponent of chaotic iterations having $x^{0}$ for initial condition is equal to $\lambda\left(x^{0}\right)=\ln (10)$.

Proof It is reminded that $g$ is piecewise linear, with a slop of $10\left(g^{\prime}(x)=10\right.$

where the function $g$ is differentiable). Then $\forall x \in \mathcal{L}, \lambda(x)=\lim _{n \rightarrow+\infty} \frac{1}{n} \sum_{i=1}^{n} \ln \left|g^{\prime}\left(x^{i-1}\right)\right|=$ $\lim _{n \rightarrow+\infty} \frac{1}{n} \sum_{i=1}^{n} \ln |10|=\lim _{n \rightarrow+\infty} \frac{1}{n} n \ln |10|=\ln 10$.

Remark 2 The set of initial conditions for which this exponent is not calculable is countable. This is indeed the initial conditions such that an iteration value will be a number having the form $\frac{n}{10}$, with $n \in \mathbb{N}$. We can reach such a real number only by starting iterations on a decimal number, as this latter must have a finite fractional part.

Remark 3 For a system having $\mathrm{N}$ cells, we will find, mutatis mutandis, an infinite uncountable set of initial conditions $x^{0} \in\left[0 ; 2^{\mathrm{N}}\left[\right.\right.$ such that $\lambda\left(x^{0}\right)=$ $\ln (\mathrm{N})$.

So, it is possible to make the Lyapunov exponent of our digital watermarking scheme as large as possible, depending on the number of least significant coefficients of the cover media we decide to consider. Obviously, a large Lyapunov exponent make it impossible to achieve the well-known Original Estimated Attacks [4]. 


\section{Conclusion and Future Works}

As a conclusion, we have available to us now a new quantitative property concerning our digital watermarking scheme based on chaotic iteration: its Lyapunov exponent is equal to $\ln (\mathrm{N})$, where $\mathrm{N}$ is the number of least significant coefficients of the cover media. This exponent allows to quantify the amplification of the ignorance on the exact initial condition (the media without watermark) after several iterations of the watermaking process. It illustrates the disorder generated by iterations of our watermarking process, reinforcing its chaotic nature.

Using the semiconjugacy described here, it will be possible in a future work to compare the topological behavior of chaotic iterations on $\mathcal{X}$ and $\mathbb{R}$, and to explore the topological security of the watermarking scheme using this new topology. Finally, an analogue study of the two other topologically secure schemes will be also conducted in order to compare these processes, being thus able to choose the best one according to the type of applications under consideration.

\section{References}

[1] David Arroyo, Gonzalo Alvarez, and Veronica Fernandez. On the inadequacy of the logistic map for cryptographic applications. X Reunin Espaola sobre Criptologa y Seguridad de la Informacin (X RECSI), 1:77-82, 2008.

[2] Jacques Bahi, Jean-François Couchot, and Christophe Guyeux. Steganography: a class of algorithms having secure properties. In IIH-MSP-2011, 7-th Int. Conf. on Intelligent Information Hiding and Multimedia Signal Processing, pages ***_***, Dalian, China, October 2011. To appear.

[3] Jacques M. Bahi and Christophe Guyeux. Topological chaos and chaotic iterations, application to hash functions. In WCCI'10, IEEE World Congress on Computational Intelligence, pages 1-7, Barcelona, Spain, July 2010. Best paper award.

[4] Francois Cayre, Caroline Fontaine, and Teddy Furon. Kerckhoffs-based embedding security classes for woa data hiding. IEEE Transactions on Information Forensics and Security, 3(1):1-15, 2008.

[5] Robert L. Devaney. An Introduction to Chaotic Dynamical Systems, 2nd Edition. Westview Pr., March 2003.

[6] Nicolas Friot, Christophe Guyeux, and Jacques Bahi. Chaotic iterations for steganography - stego-security and chaos-security. In SECRYPT'2011, Int. Conf. on Security and Cryptography, pages ***_***, Sevilla, Spain, July 2011. To appear.

[7] Christophe Guyeux, Nicolas Friot, and Jacques Bahi. Chaotic iterations versus spread-spectrum: chaos and stego security. In IIH-MSP'10, 6-th Int. 
Conf. on Intelligent Information Hiding and Multimedia Signal Processing, pages 208-211, Darmstadt, Germany, October 2010.

[8] David Richeson and Jim Wiseman. Chain recurrence rates and topological entropy. Topology and its Applications, 156(2):251 - 261, 2008.

[9] Yong Wang, Kwok-Wo Wong, Xiaofeng Liao, and Tao Xiang. A block cipher with dynamic s-boxes based on tent map. Communications in Nonlinear Science and Numerical Simulation, 14(7):3089 - 3099, 2009. 


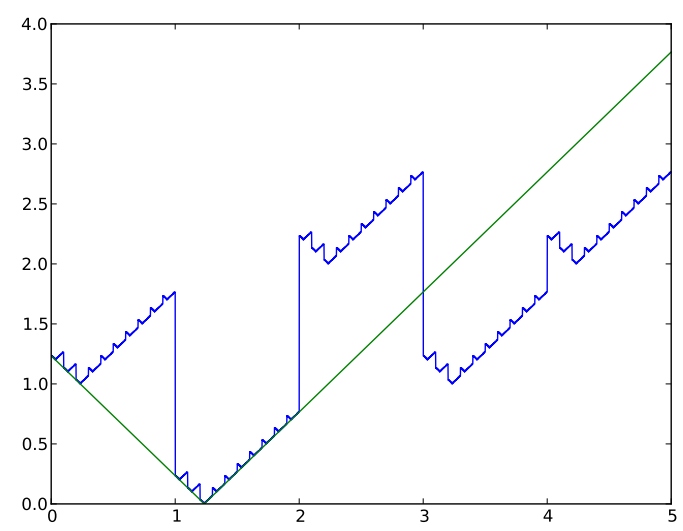

(a) Function $x \rightarrow \operatorname{dist}(x ; 1,234)$ on the interval $(0 ; 5)$.

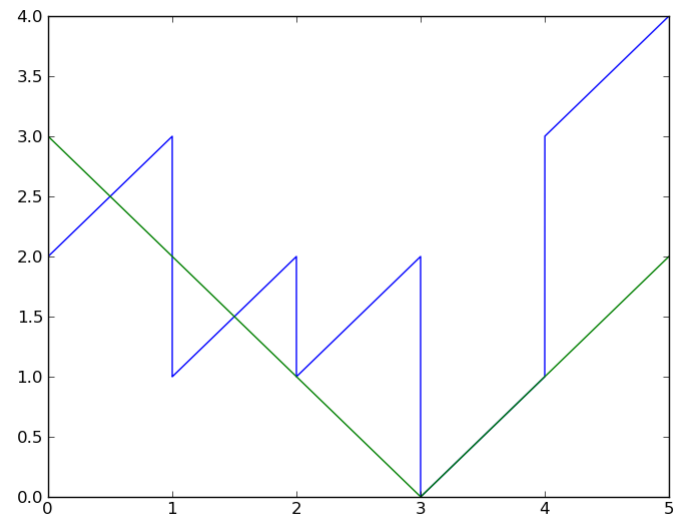

(b) Function $x \rightarrow \operatorname{dist}(x ; 3)$ on the interval $(0 ; 5)$.

Figure 1: Comparison between $D$ (in blue) and the Euclidian distance (in green). 